\title{
Effect of weekend fasting therapy on blood pressure in overweight or obese patients with hypertension
}

\author{
Jiang Jin-Peng, Zhang Geng-Peng, Wang Yuan-Yuan, Qin Jian and Li Hui* \\ Department of Traditional Chinese Medicine, Huangpu District, The First Affiliated Hospital of Sun Yat-Sen University, Guangzhou (510700), China
}

\begin{abstract}
Objective: To observe the effect of Weekend Fasting Therapy on blood pressure in overweight or obese patients with hypertension.

Methods: The 90 patients who met the obesity/overweight and hypertension diagnostic criteria were randomized and assigned into the Control group (CT), Weekend fasting group (WF) and Weekend fasting and Standardized dinner group (WFSD). Body mass Index (BMI) and blood pressure were measured before and after treatment.

Results: After 6 weeks of treatment, significantly reduced in BMI, systolic blood pressure and diastolic blood pressure in WF group and WFSD group $(P<0.05)$ but were not observed in $\mathrm{CT}$ group. BMI, blood pressure reduction, the standard and effective rate of blood pressure were more significantly changed whenever between WF group and control group $(P<0.05)$, or between WFSD group and control group $(P<0.05)$. But there was no significant differences between WF group and WFSD group. Blood pressure reduction were no correlated with the body weight, BMI, waist circumference, hip circumference, waist-to-hip ratio in WF group and WFSD group.
\end{abstract}

Conclusions: Weekend fasting therapy can reduce the blood pressure and lose weight in overweight or obese patients with hypertension.

\section{Introduction}

Hypertension is one of the most common cardiovascular diseases. As people's living standards improve and dietary patterns change, overweight and obesity have become a global trend [1-3]. It has been confirmed [4] that overweight and obesity are important risk factors for inducing hypertension. Relative to the normal population, the incidence of obese people may increase by 2 to 6 times [5]. A prospective study by Fleming showed that both systolic and diastolic blood pressures increased significantly with an increase in BMI. For every $4.5 \mathrm{~kg}$ of body weight gain, systolic blood pressure increased by $4.4 \mathrm{~mm} \mathrm{Hg}$ and diastolic blood pressure increased by $4.2 \mathrm{~mm} \mathrm{Hg}$ [6]. Due to excessive intake of high-calorie, high-fat foods at dinner, coupled with less exercise at night, excessive heat is converted into fat stored in the body, and a large amount of blood lipids are deposited on the blood vessel wall, causing atherosclerosis, thus the risk of hypertension is increased.

Fasting therapy, as a medically valuable medical practice, has been highly recognized worldwide [7]. The definition of this therapy refers to a diet therapy that prevents the treatment of certain diseases, except for the right amount of drinking water and extremely low-calorie supply, in a limited time [8]. A series of experimental studies and clinical observations have confirmed the weight-loss effect of this therapy on obese patients [9-11], and the antihypertensive effect on patients with hypertension [12-15]. In addition, weight loss and a healthy lifestyle caused by fasting will produce long-lasting antihypertensive effects [16].

Fasting methods are divided into intermittent fasting (IF) and periodic fasting (PF). Intermittent fasting includes fasting every other day or fasting twice a week. According to the working rules and eating habits of Chinese people, the intermittent fasting is arranged on the weekend, which means fasting on Saturday and Sunday, that is, "weekend fasting". Fasting on Saturday and Sunday's leisure time can both achieve the goal of treatment for the disease and avoid the harm caused by overeating on Saturday and Sunday.

In this experiment, the effects of weekend fasting therapy on blood pressure of overweight/obese patients with hypertension were studied through different dietary interventions. The effectiveness of the therapy was explored, and a new method for clinical prevention and treatment of hypertension was provided.

\section{Materials and methods}

The subjects were all the patients from the First Affiliated Hospital of Sun Yatsen University who were eligible for hypertensive patients with overweight/obesity. The diagnostic criteria were: (1) overweight and/or obesity: BMI $\geq 24.0 \mathrm{~kg} / \mathrm{m}^{2}$, waist circumference for male $\geq 85 \mathrm{~cm}$ and female $\geq 80 \mathrm{~cm}[17,18]$; (2) Patients diagnosed with hypertension and are being treated [19]. Exclusion criteria: acute malignant hypertension and hypertensive crisis; patients with secondary hypertension; abnormalities functions in heart, liver and kidney; age below 18 or over 70; pregnant women and lactating women, women with pregnancy plan within 1 year; patients with malignant tumors and blood diseases; patients with active tuberculosis; patients with ulcers and severe gastric bleeding; anorexia or excessive weight loss or malnutrition; unable

*Correspondence to: Li Hui, Attending Physician, Seventh Affiliated Hospital of Sun Yat-sen University, Chinese Medicine fasting therapy and cardiovascular disease, Republic of China, E-mail: 544252202@qq.com

Key words: weekend fasting, obesity, hypertension

Received: October 12, 2018; Accepted: October 24, 2018; Published: October 26,2018 
to cooperate with treatment, with poor compliance. The study was approved by the Ethics Committee of the First Affiliated Hospital of Sun Yatsen University, and the research process complied with the Helsinki Declaration [20]. All subjects signed informed consent.

90 eligible patients were selected and divided into Weekend fasting group (WF), Weekend fasting and standardized dinner group (WFSD) and control group according to the random number table method CT).

\section{Study method}

Weekend fasting therapy is divided into three stages: buffer period, fasting period and free diet period. The first stage: buffer period (1 day), the main purpose is to gradually control the amount of food, to transitional eliminate the psychological hunger. On the day of buffer period, eat $1500 \mathrm{~g}$ of fruit, drink about 2-3L of mineral water, and advise the subjects to perform aerobic exercise (such as walking slowly), record the amount of exercise and time, and avoid heavy physical exercise. The second stage: fasting period ( 2 days), at this stage, patients are prohibited from eating food. On the first day of fasting, dissolve $15-30$ grams of Xuan Ming powder into $500 \mathrm{ml}$ warm water to make the intestines clean. At this stage, sodium bicarbonate tablets were orally administered three times a day, $1.0 \mathrm{~g}$ ( 2 tablets) at a time; the glucurolactone tablets for 3 times, once $0.2 \mathrm{~g}$ ( 2 tablets). In addition, the subject is recommended to drink more than 3 liters of mineral water per day. Encourage the subject to have aerobic exercise. The amount of exercise and time can be recorded according to the individual's physical strength. Avoid heavy physical exercise and avoid activities that require a high concentration of energy (such as driving a vehicle). Stage 3: During the free diet (4 days), subjects were allowed to eat freely, but it is still recommended to avoid overeating. The weekend fasting + standardized dinner group (WFSD group) eats 1 bag of low-calorie nutritious meals per night (each containing $7.9 \mathrm{~g}$ protein, $2.8 \mathrm{~g}$ fat and $22 \mathrm{~g}$ starch, multivitamins and minerals, for total $200 \mathrm{Kcal}$ calories).

The above three stages need to be repeated 6 times, which will be recorded as a course of treatment; at least every two weeks, the researchers monitor their weight, blood pressure, heart rate, waist circumference and hip circumference. Drink tea, alcohol, coffee and other drinks were prohibited during treatment.

\section{Grouping plan}

A. The WF group undergo fasting therapy in accordance with standardized weekend fasting therapy procedures;

B. The WFSD group uses a standardized dinner instead of a daily dinner from Monday to Friday, except for fasting on weekends;

C. The normal diet of the CT group, without intervention, continued to follow the previous blood pressure reduction program;

\section{Observation indicators}

Main indicators: systolic blood pressure and diastolic blood pressure changes. Secondary indicators: weight, BMI, waist circumference, hip circumference, waist to hip ratio.

\section{Efficacy Criteria}

Compliance with blood pressure: According to the 2010 edition of the Guidelines for Prevention and Treatment of Hypertension in China, $\mathrm{Bp}<140 / 90 \mathrm{mmHg}$ is used as the standard value.

Rate of compliance $=$ number of blood pressure standards after taking the drug / total number of cases in the group

\section{Efficacy indicators}

Effective: After the reduction or treatment, the drug reduction standard has not been reached, but $\mathrm{Bp} \leq 130 / 85 \mathrm{mmHg}$

\section{Invalid: Bp $>130 / 85 \mathrm{mmHg}$}

Drug reduction standard: $\mathrm{Bp} \leq 120 / 80 \mathrm{mmHg}$ or after treatment, refer to the 2002 Guidelines for Clinical Research of New Drugs in Traditional Chinese Medicine which achieve significant or effective effect.

Significant effect, the diastolic blood pressure decreased by $\geq 10$ $\mathrm{mm} \mathrm{Hg}$ after treatment and fell to the normal range; or the diastolic blood pressure did not fall to normal, but it has decreased by more than $20 \mathrm{~mm} \mathrm{Hg}$, one of the two indicators reached will be regarded as the Significant effect.

Effective effect Although the diastolic blood pressure drop after effective treatment has not reached $10 \mathrm{~mm} \mathrm{Hg}$, it has dropped to the normal range; or decreased by $10-19 \mathrm{~mm} \mathrm{Hg}$. If it is systolic hypertension, the systolic blood pressure drops by $\geq 30 \mathrm{~mm} \mathrm{Hg}$.

Effectiveness $=$ number of valid cases $/$ total number of cases in the group

\section{Principle of reducing medicine (adjusted according to blood pressure)}

- If you take more than 2 kinds of antihypertensive drugs, deactivate one of them

- If you take 1 antihypertensive drug, the dose is halved

- If the blood pressure is completely normal, you do not need to take the medicine

\section{Measurement method Blood pressure}

The subjects were quietly rested for 10 minutes. The blood pressure of the right hand was measured by a mercury sphygmomanometer. The upper arm of the study object was level with the heart, the palms were laid flat, the legs were closed and the whole body was relaxed, and the cuff was under the airbag. The edge is $2-3 \mathrm{~cm}$ above the elbow. The first sound of Korotkoff is systolic blood pressure (SBP), and the fifth sound is diastolic blood pressure (DBP). The measurement is performed twice continuously, each time interval is $10 \mathrm{~min}$, and the average value is taken. If the difference between the two measurement results is large (more than $5 \mathrm{mmHg}$ ), then measure again [21]. Body weight: use a regularly calibrated TANITA MC- 180 body fat meter to measure. After urinating in the morning on an empty stomach, the subjects took off their shoes, only wore thin clothes, stepped on the weighing plate, and waited for 5 seconds before leaving the weighing plate. The staff reads the numbers on the display and weighs exactly $0.1 \mathrm{~kg}$. Waist circumference [22]: After emptying the abdominal urination, the subject was erect, and the feet were separated by $30-40 \mathrm{~cm}$. An inelastic, minimum scale of $1 \mathrm{~mm}$ was placed on the anterior superior iliac spine and connected to the lower edge of the twelfth bone. The midpoint (usually the natural narrowest part of the waist), around the abdomen in the horizontal direction, makes it close and does not oppress the skin, measuring the waist at the end of normal exhalation, the reading is accurate to $1 \mathrm{~mm}$. Hip circumference: After urinating in the morning on an empty stomach, the legs are erected upright, the arms are naturally drooping, and the tape measure is placed horizontally on the pubic symphysis and the most prominent part of the gluteus maximus. The reading is accurate to $1 \mathrm{~mm}$. 


\section{Statistical methods}

Normal distribution measurement data are expressed as mean \pm standard deviation $(\overline{\mathrm{X}} \pm \mathrm{S})$. Comparison of measurement data between groups is performed by one-way analysis of variance or Kruskal-Wallis method. The pairwise test is used to compare the differences between groups. Comparisons between groups were made using the LSD-t test or the Bonferroni method. The count data was expressed as a percentage, and the comparison between groups was performed by 2 tests; the correlation between the two variables was Pearson-related or Spearman rank correlation; the data was statistically processed using SPSS 19.0, and all statistical tests were performed using a two-sided test with a value less than 0.05 . There will be a significant difference in the statistical test when $P$ value is less than 0.05 .

\section{Results}

\section{General information}

There were no significant differences in age, body weight, body mass index, systolic blood pressure, diastolic blood pressure, waist circumference, hip circumference, and waist-to-hip ratio between the three groups $(P>0.05)$, which were comparable (Table 1$)$.

\section{Effects on blood pressure, weight, waist circumference and hip circumference of patients}

Through observation, the systolic blood pressure and diastolic blood pressure in the WF group and the WFSD group were significantly lower than before, and the difference was statistically significant $(P<0.05)$. However, there was no significant change in the control group before and after treatment. By comparison between groups, it was found that the three groups had significant differences in blood pressure $(P<0.05)$. Compared with CT group, the changes of systolic blood pressure and diastolic blood pressure in WF group and WFSD group were significantly lower than those in CT group, the difference was statistically significant $(P<0.05)$, but there was no significant difference between WF group and WFSD group (Table 2).
Through observation, the body weight and BMI of the WF group and the WFSD group were significantly lower than before, and the difference was statistically significant $(P<0.05)$, but there was no significant change before and after treatment in the control group. Through comparison between groups, it was found that the three groups had significant differences in body weight and BMI $(P<0.05)$. Compared with the CT group, the changes in body weight and BMI of the WF group and the WFSD group were significantly lower than those of the CT group, and the difference was statistically significant $(P<0.05)$, but there was no significant difference between the WF group and the WFSD group (Table 3 ).

Through observation, the waist circumference, hip circumference and waist-to-hip ratio of the WF group and the WFSD group were significantly lower than the previous ones, and the difference was statistically significant $(P<0.05)$. However, there was no significant change before and after treatment in the control group. Through comparison between groups, it was found that there were significant differences in the effects of waist circumference, hip circumference and waist-to-hip ratio among the three groups $(P<0.05)$. Compared with the $\mathrm{CT}$ group, the changes of waist circumference, hip circumference and waist-hip ratio in the WF group and the WFSD group were significantly lower than those in the CT group, and the difference was statistically significant $(P<0.05)$, but there was no difference between the WF group and the WFSD group. Significant differences (Table 4).

\section{The compliance rate and efficiency of blood pressure after treatment}

Three groups of blood pressure compliance rates were conducted $\chi^{2}$ test $\left(\chi^{2}=15.771, P<0.05\right)$, indicating significant differences between the three groups. Comparing any two groups, it can be seen that the blood pressure compliance rate of WF group and WFSD group is significantly higher than that of $\mathrm{CT}$ group $(P<0.05)$; however, there is no significant difference between WF group and WFSD group (Table 5).

Three groups of blood pressure effectiveness were conducted $\chi^{2}$ test $\left(\chi^{2}=21.667, P<0.01\right)$, indicating significant differences between

Table 1. Comparison of basic characteristics of three groups $(\overline{\mathrm{X}} \pm S)$

\begin{tabular}{|c|c|c|c|c|c|c|c|c|}
\hline Group & Age & Weight $/ \mathrm{kg}$ & $\mathrm{BMI} / \mathrm{kg} \cdot \mathrm{m}^{2}$ & $\mathrm{SBP} / \mathrm{mmHg}$ & $\mathrm{DBP} / \mathrm{mmHg}$ & $\begin{array}{c}\text { Waist } \\
\text { circumference } / \mathrm{cm}\end{array}$ & $\begin{array}{l}\text { Hip circumference } \\
\text { /cm }\end{array}$ & Waist to hip ratio \\
\hline WF & $51.6 \pm 8.7$ & $73.8 \pm 11.3$ & $27.1 \pm 3.6$ & $145.4 \pm 18.7$ & $97.0 \pm 5.2$ & $91 . .5 \pm 11.1$ & $102.2 \pm 5.8$ & $0.88 \pm 0.15$ \\
\hline WFSD & $50.2 \pm 13.7$ & $71.5 \pm 16.7$ & $28.6 \pm 4.2$ & $149.7 \pm 17.3$ & $95.3 \pm 4.8$ & $93.3 \pm 9.6$ & $104.7 \pm 6.5$ & $0.90 \pm 0.06$ \\
\hline $\mathrm{CT}$ & $52.9 \pm 7.1$ & $71.3 \pm 19.4$ & $27.8 \pm 3.8$ & $147.8 \pm 12.9$ & $94.6 \pm 3.7$ & $91.8 \pm 13.6$ & $101.0 \pm 11.7$ & $0.89 \pm 0.10$ \\
\hline
\end{tabular}

$\mathrm{n}=30 ; 1 \mathrm{mmHg} \approx 0.133 \mathrm{KPa}$; WF-weekend fasting group; WFSD- weekend fasting + standardized dinner group; CT- normal diet group; BMI- body Mass Index; SBP- systolic blood pressure DBP- diastolic blood pressure

Table 2. Effects of three groups of patients on blood pressure before and after treatment $(\overline{\mathrm{X}} \pm S)$

\begin{tabular}{|c|c|c|c|c|c|c|}
\hline Group & $\begin{array}{l}\text { Systolic pressure before } \\
\text { treatment/mmHg }\end{array}$ & $\begin{array}{l}\text { Systolic blood pressure } \\
\text { after treatment } / \mathrm{mmHg}\end{array}$ & $\begin{array}{c}\text { Systolic pressure } \\
\text { changes value } / \mathrm{mmHg}\end{array}$ & $\begin{array}{c}\text { Diastolic blood pressure } \\
\text { before treatment/ } \\
\text { mmHg }\end{array}$ & $\begin{array}{l}\text { Diastolic blood pressure } \\
\text { after treatment } / \mathrm{mmHg}\end{array}$ & $\begin{array}{l}\text { Diastolic blood pressure } \\
\text { changes value/mmHg }\end{array}$ \\
\hline WF & $145.4 \pm 18.7$ & $124.1 \pm 13.7^{*}$ & $21.3 \pm 10.3^{\#}$ & $97.0 \pm 5.2$ & $88.3 \pm 4.8^{*}$ & $8.7 \pm 1.3^{\#}$ \\
\hline WFSD & $149.7 \pm 17.3$ & $127.1 \pm 14.9^{*}$ & $22.6 \pm 10.6^{\#}$ & $95.3 \pm 4.8$ & $86.1 \pm 6.3 *$ & $9.2 \pm 1.8^{\#}$ \\
\hline $\mathrm{CT}$ & $147.8 \pm 12.9$ & $145.2 \pm 13.7$ & $2.6 \pm 0.49$ & $94.6 \pm 3.7$ & $93.9 \pm 2.6$ & $0.7 \pm 0.04$ \\
\hline
\end{tabular}

Compared with the same group before treatment, ${ }^{*} P<0.05$; Compare with $\mathrm{CT}$ group ${ }^{\#} P<0.05$

Table 3. Effects of three groups of patients on body weight and BMI before and after treatment $(\overline{\mathrm{X}} \pm S)$

\begin{tabular}{|c|c|c|c|c|c|c|}
\hline Group & $\begin{array}{l}\text { Weight before treatment/ } \\
\mathrm{kg}\end{array}$ & Weight after treatment $/ \mathrm{kg}$ & Weight change value $/ \mathrm{kg}$ & $\begin{array}{l}\text { BMI before treatment/ } \\
\qquad \mathrm{kg} \cdot \mathrm{m}^{2}\end{array}$ & $\begin{array}{l}\text { BMI after treatment/ } \\
\text { kg. } \mathrm{m}^{2}\end{array}$ & BMI change value $/ \mathrm{kg} \cdot \mathrm{m}^{2}$ \\
\hline WF & $73.8 \pm 11.3$ & $69.2 \pm 10.6^{*}$ & $4.60 \pm 2.78^{\#}$ & $27.1 \pm 3.6$ & $25.3 \pm 3.3^{*}$ & $1.82 \pm 1.02^{\#}$ \\
\hline WFSD & $71.5 \pm 16.7$ & $66.9 \pm 14.1^{*}$ & $4.61 \pm 2.47^{\#}$ & $28.6 \pm 4.2$ & $26.8 \pm 3.9^{*}$ & $1.84 \pm 0.92^{\#}$ \\
\hline $\mathrm{CT}$ & $71.3 \pm 19.4$ & $71.8 \pm 18.8$ & $-0.48 \pm 0.007$ & $27.8 \pm 3.8$ & $27.9 \pm 4.1$ & $-0.10 \pm 0.005$ \\
\hline
\end{tabular}

Compared with the same group before treatment, ${ }^{~} P<0.05$; compare with $\mathrm{CT}$ group ${ }^{\sharp} P<0.05$ 
the three groups. Comparing any two groups, it can be seen that the blood pressure reduction efficiency of WF group and WFSD group is significantly higher than that of CT group $(P<0.01)$; however, there is no significant difference between WF group and WFSD group (Table 5).

\section{Correlation analysis between blood pressure changes and changes in body weight, BMI, waist circumference, hip circumference and waist-hip ratio}

The correlation between blood pressure changes and body weight, BMI, waist circumference, hip circumference and waist-to-hip ratio in WF group and WFSD group was analyzed. It was found that there was no statistically significant correlation between blood pressure changes and the above indicators $(P>0.05)$ (Table 6).

\section{Discussion}

Hypertension is a major problem worldwide. In 2013, the World Health Organization took the prevention and control of hypertension for the first time as the theme of World Health Day, emphasizing the need to improve the control rate and treatment rate of hypertension. Overweight and obesity, as important risk factors for the onset of hypertension, are increasingly attracting human attention. Fasting therapy reduces weight loss and reduces the risk of cardiovascular disease associated with obesity by limiting the total daily calorie intake.

One of the characteristics of obese patients with hypertension is endothelial dysfunction [23]. By secreting nitric oxide and endothelin, the vascular endothelium is involved in the regulation of blood pressure. When vascular endothelial function is impaired, it releases less nitric oxide, increases endothelin, contracts small blood vessels, and increases peripheral resistance, which increases blood pressure. Previous studies have confirmed that a low-calorie diet can improve endothelial function in obese hypertensive patients [24]. Our previous study by the research team also confirmed that vascular endothelin can be significantly decreased after fasting, and nitric oxide is significantly increased, thereby lowering blood pressure [25]. Caloric restriction can increase nitric oxide synthesis by activating AMP-activated protein kinase, phosphatidylinositol 3-kinase, and protein kinase B [26]. Caloric restriction also promotes the synthesis of adiponectin, down-regulating gene expression of renin-angiotensin converting enzyme and renin type $1 \mathrm{~A}$ receptor, thereby lowering blood pressure [27]. In addition, during fasting, the plasma osmotic pressure decreases, the stimulation of osmotic pressure receptors is reduced, the release of vasopressin is reduced, the re-absorption of water in the distal convoluted tubules and collecting tubes is reduced, the amount of urination is increased, and blood pressure is lowered by reducing blood volume.

By analyzing the correlation between blood pressure changes and changes in body weight, BMI, waist circumference, hip circumference, and waist-to-hip ratio, there is no significant correlation between changes in blood pressure and changes in the above indicators, suggesting that fasting therapy has an independent antihypertensive effect. This indicates that fasting therapy can not only reduce the risk factors of cardiovascular disease, but also lower blood pressure by improving vascular endothelial function, promoting adiponectin synthesis and lowering plasma osmotic pressure, thereby delaying the process of atherosclerosis and reducing damage to the target organ.

The results of this study show that although taking antihypertensive drugs alone can reduce the blood pressure of patients with hypertension, but not all of them meet the standard; while performing weekend fasting for 6 weeks on the basis of taking antihypertensive drugs, not only can reduce the weight of patients, but also improve the compliance rate and efficiency of blood pressure control, and even achieve the purpose of reducing medicine. This suggests that caloric restriction contributes to a further reduction in blood pressure in hypertensive patients. The results of this study are consistent with previous experimental

Table 4. Effect of waist circumference, hip circumference and waist-to-hip ratio before and after treatment in three groups of patients ( $\overline{\mathrm{X}} \pm S$ )

\begin{tabular}{|c|c|c|c|c|c|c|c|c|c|}
\hline Group & $\begin{array}{c}\text { Waist } \\
\text { circumference } \\
\text { before } \\
\text { treatment } / \mathrm{cm}\end{array}$ & $\begin{array}{c}\text { Waist } \\
\text { circumference } \\
\text { after treatment } \\
/ \mathrm{cm}\end{array}$ & $\begin{array}{c}\text { Waist } \\
\text { circumference } \\
\text { change value } \\
/ \mathrm{cm}\end{array}$ & $\begin{array}{c}\text { Hip } \\
\text { circumference } \\
\text { before } \\
\text { treatment } / \mathrm{cm}\end{array}$ & $\begin{array}{c}\text { Hip } \\
\text { circumference } \\
\text { after treatment } \\
/ \mathrm{cm}\end{array}$ & $\begin{array}{c}\text { Hip } \\
\text { circumference } \\
\text { change value/cm }\end{array}$ & $\begin{array}{l}\text { Waist to hip } \\
\text { ratio before } \\
\text { treatment }\end{array}$ & $\begin{array}{l}\text { Waist to hip } \\
\text { ratio before } \\
\text { treatment }\end{array}$ & $\begin{array}{c}\text { Waist to hip } \\
\text { ratio } \\
\text { Change value }\end{array}$ \\
\hline WF & $91.5 \pm 11.1$ & $84.8 \pm 12.3^{*}$ & $6.71 \pm 3.61^{\#}$ & $102.2 \pm 5.8$ & $97.8 \pm 6.1^{*}$ & $4.40 \pm 1.88^{\#}$ & $0.88 \pm 0.15$ & $0.85 \pm 0.09^{*}$ & $0.03 \pm 0.012^{\#}$ \\
\hline WFSD & $93.3 \pm 9.6$ & $86.4 \pm 12.5^{*}$ & $6.9 \pm 3.70^{\#}$ & $104.7 \pm 6.5$ & $100.9 \pm 5.3 *$ & $3.82 \pm 1.03^{\#}$ & $0.90 \pm 0.06$ & $0.87 \pm 0.32 *$ & $0.03 \pm 0.026^{\#}$ \\
\hline $\mathrm{CT}$ & $91.8 \pm 13.6$ & $92.3 \pm 10.5$ & $-0.5 \pm 0.015$ & $101.0 \pm 11.7$ & $101.3 \pm 9.4$ & $-0.3 \pm 0.012$ & $0.89 \pm 0.10$ & $0.90 \pm 0.01$ & $-0.01 \pm 0.008$ \\
\hline
\end{tabular}

Compared with the same group before treatment, ${ }^{*} P<0.05$; compare with $\mathrm{CT}$ group ${ }^{\#} P<0.05$

Table 5. Blood Pressure Compliance Rate and Efficiency of Three groups of patients

\begin{tabular}{|c|c|c|c|c|c|c|c|}
\hline \multirow{2}{*}{ Group } & \multirow{2}{*}{ Cases } & \multicolumn{2}{|c|}{ Compliance } & \multicolumn{2}{|c|}{ Valid } & \multicolumn{2}{|c|}{ Invalid } \\
\hline & & Cases & Rate (\%) & Cases & Rate (\%) & Cases & Rate (\%) \\
\hline WF & 30 & 27 & $90^{\#}$ & 24 & $80^{\# \#}$ & 6 & 20 \\
\hline WFSD & 30 & 29 & $96.67^{\#}$ & 25 & $83.33^{\# \#}$ & 5 & 16.67 \\
\hline $\mathrm{CT}$ & 30 & 20 & 66.67 & 4 & 13.33 & 26 & 86.67 \\
\hline$P$ Value & & \multicolumn{2}{|c|}{$<0.05$} & \multicolumn{2}{|c|}{$<0.01$} & & \\
\hline
\end{tabular}

Compare with CT group ${ }^{*} P<0.05,{ }^{\#} P<0.01$

Table 6. Correlation coefficient between blood pressure change and body weight, BMI, waist circumference, hip circumference, waist-hip ratio (value of $r$ )

\begin{tabular}{|c|c|c|c|c|c|c|}
\hline Group & Index & Weight & BMI & Waist circumference & Hip circumference & Waist to hip ratio \\
\hline \multirow{2}{*}{ WF } & Systolic blood pressure & 0.179 & 0.175 & 0.211 & 0.017 & 0.168 \\
\hline & Diastolic blood pressure & 0.293 & 0.238 & 0.084 & 0.212 & 0.092 \\
\hline \multirow{2}{*}{ WFSD } & Systolic blood pressure & 0.17 & 0.201 & 0.182 & 0.02 & 0.187 \\
\hline & Diastolic blood pressure & 0.11 & 0.15 & 0.07 & 0.16 & 0.101 \\
\hline
\end{tabular}


studies and clinical observations of caloric restriction on weight loss in obese patients [9-11] and antihypertensive effects in patients with hypertension [12-15].

In clinical observation, we found that although there was no significant difference between the WFSD group and the WF group in terms of pressure reduction amplitude, blood pressure reduction efficiency, compliance rate and general indicators, it had a better trend than the WF group. Therefore, considering the WFSD group may have a better effect on the buck. The test results may be related to the small number of samples in this study and the poor compliance of patients in the dining package.

The shortcoming of this study is that most of the patients collected were mild and moderate hypertensive patients, while there were fewer cases of severe hypertension and hypertensive emergencies. Therefore, this study is more important for the treatment of overweight/obesity with mild to moderate hypertension. Combined with the current situation in China, the majority of patients with hypertension are mild and moderate hypertension (90\%), and mild hypertension accounts for more than $60 \%$ [28]. Fasting therapy has a benign antihypertensive effect $[29,30]$, and its decompression amplitude is closely related to the basal blood pressure value but has no excessive reduction effect on normal blood pressure. Therefore, this therapy is safe and suitable for most people with high blood pressure.

\section{Conclusion}

At present, when selecting antihypertensive drugs, the firstline drugs recommended according to the international treatment guidelines are combined with cardiovascular risk factors and complications of patients, and individualized programs are selected. For patients with overweight/obesity and hypertension, this study provides new treatment ideas and guidelines that is to combine antihypertensive drugs with weekend fasting, which can reduce weight, lower blood pressure, and reduce the amount and type of medication, delay the progression of atherosclerosis, reduce target organ damage, and also reduce adverse drug reactions, reduce the economic burden of patients, thereby achieving the best therapeutic effect. At the same time, weekend fasting is suitable for the characteristics of Chinese office workers. It is easier to be accepted by the public to perform fasting during the leisure time on Saturday and Sunday. Moreover, this method is easy to operate, safe and low in cost, which is worthy for clinical promotion and application.

\section{Fund project}

Shenzhen Health and Family Planning System Research Project (SZSW2018001); Shenzhen Health and Family Planning System Research Project (SZLY2018002).

\section{References}

1. Roth J, Qiang X, Marban SL, Redelt H, Lowell BC (2004) The obesity pandemic: where have we been and where are we going? Obes Res 12: 88S-101S. [Crossref]

2. Popkin BM, Adair LS, Ng SW (2012) Global nutrition transition and the pandemic of obesity in developing countries. Nutr Rev 70: 3-21. [Crossref]

3. Swinburn BA, Sacks G, Hall KD, McPherson K, Finegood DT, et al. (2011) The global obesity pandemic: shaped by global drivers and local environments. Lancet $378: 804$ 814. [Crossref]

4. Pan Yang-zhong (2002) Epidemiological Study on Overweight-obesity and the Relation of It with Hypertension and Other Related Factors in Population under Pr evention and Cure for Cerebral-cardiovascular Diseases in Guizhou Township. Chin J Prev Contr Chron Non-commun Dis 10: 197- 201.
5. Wang Zi-kuan, Cheng Zhi-qing (2004) The progression of epidemiological study on Overweight-obesity and hypertension population. Modern Trad Chin Med 3: 19-21.

6. Ma Wen-jun, Xu Yan-jun, Guo Runing (2002) The progression of epidemiological study on Overweight-obesity population. Foreign Med 19: 127-131.

7. Fahrner H (1991) Die Fastenkur. Arztezeitschrift fur Naturheilverfahren 7: 544-548.

8. Ke bin, Qin Jian, Meng Jun (2009) The progression study of fasting therapy. Shenzhen Zhongxiyi Jiehe Zazhi 19: 55.

9. Varady KA, Bhutani S, Church EC, Klempel MC (2009) Short-term modified alternateday fasting: a novel dietary strategy for weight loss and cardioprotection in obese adults. Am J Clin Nutr 90: 1138-1143. [Crossref]

10. Klempel MC, Kroeger CM, Varady KA (2013) Alternate day fasting (ADF) with a high-fat diet produces similar weight loss and cardio-protection as ADF with a low-fat diet. Metabolism 62: 137-143. [Crossref]

11. Bhutani S, Klempel MC, Kroeger CM, Trepanowski JF, Varady KA (2013) Alternate day fasting and endurance exercise combine to reduce body weight and favorably alter plasma lipids in obese humans. Obesity (Silver Spring) 21: 1370-1379. [Crossref]

12. Goldhamer AC, Lisle DJ, Sultana P, Anderson SV, Parpia B, et al. (2002) Medically supervised water-only fasting in the treatment of borderline hypertension. J Altern Complement Med 8: 643-650. [Crossref]

13. Schwartz MW, Seeley RJ (1997) Seminars in medicine of the Beth Israel Deaconess Medical Center. Neuroendocrine responses to starvation and weight loss. $N$ Engl J Med 336: 1802-1811. [Crossref]

14. Muller H, Wilhelmi de Toledo F, Schuck P (2001) Blutdrucksenkung durch Fasten bei adiposen und nichtadiposen Hypertonikern. Perfusion 14: 108-112.

15. Dessi-Fulgheri P, Sarzani R, Serenelli M, Tamburrini P, Spagnolo D, et al. (1999) Low calorie diet enhances renal, hemodynamic,and humoral effects of exogenous atrial natriuretic peptide in obese hypertensives. Hypertension 33: 658-662. [Crossref]

16. Michalsen A, Li C (2013) Fasting Therapy for Treating and Preventing Disease Current State of Evidence. Forsch Komplementmed 20: 444-453. [Crossref]

17. Coorperative Meta-analysis Group of China Obesity Task Force (2002) Predictive values of body mass index and waist circumference to risk factors of related diseases in Chinese adult population. Chin J Epidemiol 23: 5-10.

18. Coorperative Meta-analysis Group of Working Group on Obesity in China (2002) Prospective study for cut-off points of body mass index in Chinese adults. Chin $J$ Epidemiol 23: 431-434.

19. Writing group of 2010 Chinese guidelines for the management of hypertension (2011) 2010 Chinese guidelines for the management of hypertension. Chin J Hypertens 19: 701-743.

20. World Medical Association (2002) World Medical Association Declaration of Helsinki:ethical principles for medical research involving human subjects. JAMA 310 : 2191-2194. [Crossref]

21. Holecki M, Dulawa J, Chudek J (2012) Resistant Hypertension in Visceral Obesity. Eur J Intern Med 23: 643-648. [Crossref]

22. Calloway DH, Calloway CW, Chumlea WC (1998) Anthropometric standardization reference manual. Champaign IL: Human Kinetics 39-54.

23. Ding Xiao-shuang (2001) Guizhi Fuling pills on the role of vascular endothelial injury in spontaneously hypertensive rats. Foreign Med 18: 113-118.

24. Sasaki S, Higashi Y, Nakagawa K, Kimura M, Noma K, et al. (2002) A low-calorie diet improves endothelium-dependent vasodilation in obese patients with essential hypertension. Am J Hypertens 15: 302-309. [Crossref]

25. Ke Bin, Shi Lin, Zhang Jun-Jie (2012) Modified Linggui Shugan decoction could protect the Vascular endothelial of phlegm-dampness due to spleen deficiency hyperlipidemia patients. Chin J Tradit Med Sci Technol 19: 207-210.

26. García-Prieto CF, Pulido-Olmo H, Ruiz-Hurtado G, Gil-Ortega M, Aranguez I, et al. (2015) Mild caloric restriction reduces blood pressure and activates endothelial AMPK-PI3K-Akt-eNOS pathway in obese Zucker rats. Vascul Pharmacol 65: 3-12. [Crossref]

27. Takatsu M, Nakashima C, Takahashi K, Murase T, Hattori T, et al. (2013) Calorie restriction attenuates cardiac remodeling and diastolic dysfunction in a rat model of metabolic syndrome. Hypertension 62: 957-965. [Crossref]

28. He J, Gu D, Wu X, Reynolds K, Duan X, et al. (2005) Major causes of death men and women in China. N Engl J Med 35: 1124-1134. [Crossref] 
Jin-Peng J (2018) Effect of weekend fasting therapy on blood pressure in overweight or obese patients with hypertension

29. Goldhamer A, Lisle D, Parpia B, Anderson SV, Campbell TC (2001) Medically Supervised Water-only Fasting in the Treatment of Hypertension. Manipulative Physiol Ther 24: 335-359. [Crossref]
30. Goldhamer AC, Lisle DJ, Sultana P, Anderson SV, Parpia B, et al. (2002) Medically supervised water-only fasting in the treatment of borderline hypertension. J Altern Complement Med 8: 643-650. [Crossref]

Copyright: (C2018 Jin-Peng J. This is an open-access article distributed under the terms of the Creative Commons Attribution License, which permits unrestricted use, distribution, and reproduction in any medium, provided the original author and source are credited. 